\title{
Factores asociados a la toma de decisiones sobre el presupuesto colombiano
}

\section{Factors Associated to the Decision Making of Colombian Budget}

Jaime Abel Huertas ${ }^{1}$

Universidad Nacional de Colombia, Departamento de Estadística, Bogotá, Colombia. jahuertasc@unal.edu.co

https://orcid.org/0000-0002-4981-9848

\section{Adriana Francisca Salinas ${ }^{2}$}

Pontificia Universidad Javeriana, Facultad de Ciencias Políticas, Bogotá, Colombia. adriana-salinas@javeriana.edu.co

https://orcid.org/0000-0002-4981-9848

2 Doctora en Economía. 


\section{Resumen}

El presente artículo propone un análisis de los debates hechos por los miembros de las Comisiones Económicas del Congreso de Colombia, sobre el Presupuesto General de la Nación de 2002 a 2009, para determinar si las decisiones sobre este han sido tomadas con racionalidad acotada. La base teórica son los planteamientos sobre la toma de decisiones realizados por Lindblom (1959), quien asume el concepto de racionalidad acotada de los agentes propuesto por Simon (1957). La información de las sesiones se analiza con un modelo donde el debate, como variable dependiente, es explicado con covariables relacionadas a las características de los parlamentarios. Con base en un modelo estadístico longitudinal con retiros informativos y covariables dependientes en el tiempo, encontramos factores asociados al debate que nos permiten concluir que las decisiones no fueron tomadas con absoluta racionalidad técnica.

Palabras clave: economía política; presupuesto nacional; comités decisores; análisis longitudinal; modelamiento conjunto.

Clasificación JEL: H61, H30, N46, C33.

\section{Abstract}

The following paper proposes an analysis of the debates made by the members of the Economic Commissions of the Congress of Colombia on the General Government Budget from 2002 to 2009, to determine if the decisions on this have been taken with bounded rationality. The theoretical basis are the approaches to decision making made by Lindblom (1959), who assumes the concept of bounded rationality of the agents proposed by Simon (1957). The information of the sessions is analyzed through a model where debate, as a dependent variable, is explained with covariates related to the characteristics of the members of the parliament. Based on a longitudinal statistical model with informative drop-out and time-dependent covariates, the found factors associated to the debate allowed us to conclude that the decisions were not taken with any absolute technical rationality.

Keywords: Political economy; National budget; Committee decision making; Longitudinal analysis; Joint modelling.

¿Cómo citar este artículo? / How to quote this article?

Huertas, J. A. y Salinas A. F. (2019). Factores asociados a la toma de decisiones sobre el presupuesto colombiano. Sociedad y economía, (38), 45-63. https://doi.org/10.25100/sye.voi38.8025 


\section{Introducción}

En Colombia, el proceso decisorio sobre el Presupuesto General de la Nación (abreviadamente Presupuesto), comienza con la formulación del proyecto de ley por el Ministerio de Hacienda, quien lo presenta a las Comisiones Económicas Conjuntas del Congreso (terceras y cuartas de Cámara y Senado), para que sea debatido y aprobado en una primera fase. Superada la primera fase, donde se surten las primeras modificaciones al proyecto, es pasado a plenarias del Congreso para su discusión y aprobación final. Las Comisiones Económicas, al igual que otras comisiones legislativas, tienen una función de análisis técnico y a profundidad de los temas que les competen.

En este artículo se presenta un análisis mediante modelos estadísticos sobre el proceso decisorio del Presupuesto en la fase legislativa de las Comisiones Económicas Conjuntas. El propósito de la modelación es determinar si hay asociaciones entre la decisión de aprobación del Presupuesto y algunas características de los parlamentarios, que puedan identificarse con las propuestas teóricas de Simon (1957) y Lindblom (1959).

Una característica fundamental en el análisis de la toma de decisiones en economía es el supuesto de racionalidad de los agentes, que se basa en información completa. En contravía, teóricos como Lindblom (1959) trabajaron sobre la toma de decisiones económicas en contextos políticos, asumiendo el concepto de racionalidad acotada de Simon (1957), quien propone que dicha limitación puede darse por dos vías: por ausencia de información, y por limitaciones de conocimiento y de capacidad de cálculo del decisor. Lindblom (1959) también plantea otros elementos como el ajuste mutuo partidario para llegar a una decisión final vía acuerdos. Al cumplirse 50 años de la publicación del artículo de Lindblom (1959), varias universidades de Canadá realizaron un examen de la actualidad de su modelo, encontrando que es uno de los autores más relevantes en investigación social. La revisión, publicada en la revista Policy and Society del año 2011, concluyó la vigencia de partes de sus postulados (Salinas y Jalil, 2014).

En la literatura de tipo empírico sobre el análisis de toma de decisiones en comités, se encuentra que existen modelos econométricos diseñados principalmente para comisiones de política monetaria. Un modelo referente es el de Chappell, McGregor y Vermileya (2005), que analiza la influencia de los miembros del Comité Federal de Mercado Abierto (FOMC) del Sistema de Reserva Federal (FED) de los Estados Unidos, en la adopción de la tasa de interés de referencia. El estudio propone un modelo donde la variable dependiente se define de forma binaria basada en la decisión que toman los miembros del comité de adoptar o no la tasa de interés sugerida por la presidencia del FOMC. Tal respuesta, modelada con las características de los agentes decisores, encontró asociación con algunas de ellas, como el partido político. Aunque las decisiones tomadas por comités de análisis de presupuestos son diferentes a las de política monetaria, el modelo que planteamos para nuestro problema es algo similar al propuesto por Chappell et al. (2005); pero, a diferencia de este, no utilizamos la votación como la variable dependiente, sino el debate en la comisión.

Nuestro modelo analiza las actas de las sesiones de las Comisiones Económicas de 2002 a 2009 (dos periodos legislativos). De ellas se tenía planificado extraer las votaciones individuales de aprobación del proyecto del Presupuesto modificado en las sesiones. Desafortunadamente no fue posible obtener su registro completo. Escogimos entonces como variable dependiente para los modelos, una que refleje la posición de cada parlamentario sobre la propuesta inicial del gobierno. Esa variable es el "debate en contra del proyecto del Presupuesto", pues por principio profesional y técnico, debería concluir en no aprobación si los cambios no son realizados conforme al debate, como efectivamente sucede en la mayoría de los casos. La cuantificación de las actas de las sesiones que determina si cada parlamentario debatió o no, va acompañada de algunas de sus características. Este conjunto de datos para cada sesión conforma una base global con información dependiente del tiempo, que analizamos con mo- 
delos longitudinales estadísticos. Por tratarse de decisiones tomadas al interior de un comité técnico, los resultados del modelo serán interpretados bajo el principio de que no debería haber algunos factores asociados a la decisión de aprobación del Presupuesto. De ser así, se tendría evidencia suficiente para concluir que las decisiones no se tomaron con absoluta racionalidad técnica.

El modelo longitudinal en este trabajo lo vemos desde un punto de vista cuantitativo, complementario al análisis textual de Salinas y Huertas (2018), donde se asumió al lenguaje como una forma de verbalización de la racionalidad. El estudio encontró que el caso analizado se puede clasificar de acuerdo a las características de la racionalidad acotada desde los planteamientos teóricos de Simon (1957) y Lindblom (1959), por la detección de asimetrías de información y de limitaciones y sesgos de los parlamentarios. En particular, se detectó relación directa entre la formación educativa y el debate. Esto es, baja educación en economía implica baja calidad del debate y viceversa. Si partimos del supuesto de que solo se puede debatir el Presupuesto adecuadamente teniendo conocimientos avanzados en economía, entonces el no debate implica una evidencia de no dominio del tema del Presupuesto y, por tanto, racionalidad acotada en la decisión. Este supuesto no necesariamente es cierto, pero lo acogemos con base en un análisis presentado en la discusión. Por lo pronto mencionemos que, dado el efecto del grupo asesor que tiene asignado por ley cada parlamentario, es de esperarse que no existan mayores diferencias entre la cantidad de debates y los conocimientos en economía determinados por la profesión de los parlamentarios. Pero esa diferencia sí existe y es bien marcada. Con esto tenemos evidencia de que la profesión en economía está siendo crucial en la toma de decisiones sobre el Presupuesto.

El estudio de análisis textual de Salinas y Huertas (2018) también concluye que el proceso decisorio sobre el Presupuesto, se puede identificar con el planteamiento del incrementalismo desarticulado de Lindblom (1959). Así pues, tenemos que el análisis textual mostró una visión amplia de lo sucedido en las comisiones, pero como es evidente, solo analiza a quienes hacen discursos. En cambio, el longitudinal incluye a todos los individuos. Por esto y debido al enfoque cuantitativo del modelo longitudinal, se justifica su desarrollo y presentación en una revista especializada en economía matemática, distinta a la de enfoque político donde fue publicado el análisis textual. Podríamos haber adoptado otros tipos de análisis para el trabajo, pero los enfoques elegidos cuantitativo y cualitativo dieron buen entendimiento del problema. Estos enfoques, presentados en revistas diferentes, son el resultado de la tesis de doctorado de Salinas (2015).

\section{Normativa institucional}

El Congreso Colombiano es bicameral, integrado por el Senado (elegido por un distrito electoral nacional) y por la Cámara de Representantes (elegida por el distrito electoral regional). Los legisladores son elegidos democráticamente por un período de cuatro años, que coincide con la elección del presidente de la República. La reunión de todos los senadores y representantes se llama plenaria. Antes de la plenaria, los legisladores se reúnen en grupos llamados comisiones para estudiar y debatir profundamente temas específicos. Las propuestas iniciales debatidas y modificadas dentro de cada comisión, se socializan y se deciden finalmente en el pleno del Congreso.

Las leyes pueden ser presentadas por iniciativa de los congresistas, pero en el caso del proyecto del Presupuesto no la hay. Únicamente es el ejecutivo, en cabeza del Ministerio de Hacienda, quien debe presentar la propuesta ante el Congreso y este solo podrá proponerle enmiendas, que deberán ser aprobadas por el gobierno para ser incluidas en tal proyecto. Una vez acordadas las enmiendas, las Comisiones Económicas votan la aprobación del proyecto modificado y lo envían para ser discutido en la plenaria.

\section{Toma de decisiones en la investigación de política económica}

El análisis de toma de decisiones es un proceso que involucra conceptos de diferentes áreas del 
conocimiento de una manera interdisciplinaria (Hansson, 1994). Áreas como psicología, economía, administración de empresas, ciencia política y estadística. Algunas diferencias entre los modelos que intentan explicar el proceso de toma de decisiones vienen dadas por la forma en que cada uno de los modelos asume la racionalidad de los agentes. El modelo racional supone que las decisiones que toman los agentes pasan de forma lógica por ciertas etapas, para lograr las mejores políticas. Como una crítica al modelo racional, Herbert Simon afirmó que la racionalidad de los agentes es acotada, en referencia a las limitaciones del conocimiento y la capacidad de cálculo del decisor (Durlauf y Blume, 2008).

Lindblom (1959) comparte el concepto de racionalidad limitada de Simon, formula un modelo de toma de decisiones económicas en contextos políticos y plantea que las decisiones se toman paso a paso para reducir los riesgos de una planificación sobredimensionada. Debido a esto, el modelo de Lindblom se conoce como incrementalismo y, recientemente, como gradualismo. Lindblom también plantea otros elementos en su modelo, como el ajuste mutuo partidario para llegar a una decisión final vía acuerdos. Dentro de los primeros trabajos de aplicación del modelo incrementalista -al tema presupuestario de Estados Unidos- se tiene el realizado por Wildavsky (1964). También hay trabajos más recientes como el de Good (2011) sobre el caso presupuestario de Canadá, y el de Caamaño y Lago-Peñas (2011), para el caso español.

Los trabajos de Simon y Lindblom sobre toma de decisiones son básicamente teóricos y no modelos cuantitativos. Las propuestas econométricas están focalizadas principalmente en el análisis de decisiones en comités de política monetaria, $y$ enfatizan en las características individuales de sus miembros. El modelo de Chappell et al. (2005), que analiza la toma de decisiones del FOMC, se ha extendido al análisis de comités de política monetaria en diferentes países, debido a la similitud que tienen con el organismo de Estados Unidos para el cual fue desarrollado. A partir de los archivos de informes de las reu- niones, los autores construyen una función de reacción (regresión) para cada miembro y, con base en ella, se toma la decisión para todo el grupo. La variable dependiente representa la decisión, a favor o en contra, de las tasas de interés propuestas por la presidencia del FOMC para distintos periodos de tiempo y las independientes tienen en cuenta, entre otras, algunas características de los miembros. Los investigadores encontraron que las decisiones del FOMC están relacionadas con las preferencias de sus miembros y con las influencias políticas que cada uno de los agentes podría tener. El estudio dispone de información en el tiempo, pero sus modelos son de tipo transversal utilizando el criterio de modelo probit.

Podemos citar otros trabajos de tipo cuantitativo sobre toma de decisiones en comités donde la variable dependiente se basa en una votación, con el de Jung y Kiss (2012) y Eijffinger, Mahieu y Raes (2013). No hemos encontrado estudios para América Latina ni trabajos como el de Chappell et al. (2005) para el análisis de toma de decisiones presupuestales.

\section{Metodología de análisis \\ 4.1 Información disponible}

En la publicación oficial institucional titulada "Gaceta del Congreso", que contiene transcripciones literales de las discusiones de los miembros de las Comisiones Económicas, se obtiene la información sobre el debate. Lo referente a características de los parlamentarios como sexo, región, nivel educativo, profesión y problemas judiciales, se obtuvo de fuentes externas al Congreso disponibles en la web. Muchos de esos datos fueron obtenidos en la página web denominada "Congreso Visible" de la Universidad de los Andes que, aunque tiene bastante información, es incompleta para los propósitos del presente trabajo. Se recurre a esta estrategia debido a que las fuentes del congreso disponibles no suministran información detallada de las características de los congresistas, ni de su trabajo político. En cuanto al Congreso y la política, tenemos las siguientes variables ex- 
traídas de fuentes administrativas del Congreso: partido político, membresía a la cámara o senado, titularidad o reemplazo de un escaño y ser ponente o no de propuestas modificatorias al proyecto de Presupuesto.

\subsection{Variable dependiente}

Como las votaciones sobre el Presupuesto tienen muy mal registro, el análisis para definir el tipo de racionalidad en las decisiones tomadas en las Comisiones Económicas, lo centramos en el debate (en contra del proyecto de Presupuesto). Así, mediremos los factores asociados a una decisión, a través del "deber ser" que da el debate.

La variable dependiente es una crítica al proyecto de Presupuesto basada en discursos extensos o moderados y respaldados con cifras obtenidas de instituciones oficiales o no oficiales. La variable es de tipo Bernoulli con valores: 1 (éxito) para el debate en contra del proyecto de Presupuesto y o (fracaso) en cualquier otro caso. Por cada sesión, la variable tomará un único valor por parlamentario, así haya intervenido más de una vez, en la misma, con diferentes debates. Las posiciones a favor del proyecto fueron casi inexistentes y, por tanto, no incluidas como otro nivel dentro de la variable. Las intervenciones a favor de su diseño y de las políticas del gobierno, fueron en general muy cortas, superficiales y no documentadas. Por esto, en adelante nos referimos al debate como una posición en contra del Presupuesto.

\subsection{Covariables}

Por tratarse del análisis de las decisiones técnicas de un comité, en un ambiente político, los factores asociados para explicar la variable dependiente los tomamos del conocimiento del decisor sobre el tema analizado y de su posición en el ambiente político. Partimos de la hipótesis de que los factores que deben explicar la mayor parte de la variabilidad de la respuesta son el conocimiento, por tratarse de decisiones técnicas, y el político, por ser la naturaleza del Presupuesto de ese orden (Giraldo, 2009). Lo razonable y asequible es relacionar la posición política al partido, así como en Chappell et al. (2005), y el conocimiento al nivel educativo y la profesión.
Obviamente la política tiene muchos matices que no pueden ser explicados por un único ítem. Por esto se toman otras variables que interaccionan con los dos factores principales. Esas variables lógicamente son las relacionadas al ambiente del Congreso donde se toman las decisiones y algunas características sociodemográficas disponibles. Así, podemos diferenciar la información en tres direcciones: conocimiento, política y características sociodemográficas. Para su obtención, se extrajo todo lo posible de las fuentes de información citadas.

Conocimiento: como no fue posible obtener toda la información sobre el nivel educativo, se mezcló con la profesión en la variable "Profesión en Economía" (o simplemente "Profesión"). Como la investigación se centra en el análisis de una comisión económica, el objetivo no es conocer la profesión de cada miembro, sino saber si son economistas o no.

Política: el partido político fue categorizado en los niveles "oposición" y "coalición de gobierno". De los 32 que hicieron parte de las Comisiones Económicas solo tres hicieron oposición. Todos diferentes en su historia y esencia, pero teniendo en común que no hicieron parte de las cuotas burocráticas del gobierno. El Mira (religioso) y el Polo (izquierda) tuvieron a todos sus miembros consistentemente en la oposición, pero el Liberal (tradicional) tuvo una oposición más fuerte en el periodo 2005-2009 y no fue hecha por todos sus miembros. Para no introducir sesgos con una selección subjetiva en la determinación de quién hacía oposición y quién no en los miembros del partido LIBERAL, se decidió incluirlos a todos ellos dentro de la categoría de "oposición", siempre y cuando no hubieran cambiado de partido en el periodo de análisis. Al lector que no conozca las costumbres políticas de Colombia, esto del cambio de partido le parecerá extraño, pero es una práctica común en el país. También es común que los parlamentarios pidan ser reemplazados en algún momento durante el periodo legislativo de cuatro años, lo que generalmente ocurre al final; esta característica está incluida en el modelo con el nombre de "Reemplazo". Se incluye porque no es admisible que un tema tan complejo como el Presupuesto, pueda ser entendido y dominado en un corto periodo de reemplazo. Por otra parte, algunos parlamentarios hacen propuestas de 
enmienda al proyecto de Presupuesto y se les llama "parlamentarios ponentes". Esta característica es analizada en una variable denominada "Ponente". Las iniciativas de los ponentes inciden en el Presupuesto y pueden ser defendidas en las sesiones con debates, pero en general son individuales y no de partido. Por eso son tenidas en cuenta por aparte en el modelo.

Sociodemográficas: debido a que la sociedad colombiana se ha visto profundamente afectada por el conflicto armado en todos los aspectos, también se miden sus efectos en el proceso de determinación del Presupuesto, a través de los procesos judiciales de los congresistas. Hasta 2014, habían sido condenados 62 congresistas por sus relaciones con grupos armados ilegales y otros 125 tenían procesos judiciales por supuestos vínculos con esos grupos. También hubo 9 condenados por delitos relacionados con corrupción, para un total de 71 condenados. En el caso de las Comisiones Económicas, son 27 condenados y 24 con procesos judiciales abiertos. De las condenas, 10 fueron para sus presidentes (31 en el periodo de análisis).

Dado que los parlamentarios con problemas judiciales pertenecen principalmente a una región particular del país (departamentos del litoral Atlántico o la región del Caribe), la variable "Región" también se analiza en el modelo. Se entiende la variable "Región" por el sitio donde el parlamentario ha tenido la mayor parte de su trabajo político, que coincide generalmente con la de su nacimiento. La edad y el sexo fueron contemplados para el modelo, pero como no fue posible obtener toda la información sobre las edades, no fue incluida.

Las siguientes son las covariables con sus niveles:

- Profesión: Economista (1); Otras profesiones (2); No profesional (3).

- Partido Político: Coalición de gobierno (o); Oposición (1).

- Reemplazo: Reemplazo de una curul (o); Titular de una curul (1).
- Ponente: No ponente de proyectos (0); Ponente de proyectos (1).

- Proceso Judicial: Sin proceso (o); Convicto o con proceso judicial abierto (1).

- Región: Capital del país - Bogotá (1); Caribe (2); Otras (3).

- Sexo: Hombre (1); Mujer (o).

Las covariables medidas con variaciones en el tiempo para cada parlamentario fueron "Ponente" y "Reemplazo". No obstante, la variación fue muy poca. Por ejemplo, solo tres parlamentarios fueron titulares en un período legislativo, y reemplazantes en el otro. Algo similar ocurrió con la covariable "Ponente".

\subsection{Tipo de análisis}

La variable dependiente resume las intervenciones de cada parlamentario en una secuencia de resultados binarios dependiendo de si debatió o no en contra del Presupuesto en las sesiones. Variable que se explica con características individuales de los parlamentarios. Aun cuando resumimos la información a un tipo similar a la del estudio de Chappell et al. (2005), nosotros la analizamos con modelos longitudinales basados en modelos logit, debido a que la variable respuesta (binaria) -y algunas independientes- son tiempo-dependientes $\mathrm{y}$, por tanto, es imperativo que los modelos tengan en cuenta dicha estructura dependiente del tiempo. La adopción de un modelo longitudinal implica correr el riesgo de no cumplir varios supuestos que los modelos transversales no tienen. Un supuesto muy importante es que no existan retiros informativos. Esto es la no obtención de toda la cadena de información programada para cada individuo debido a patrones que no son al azar. En nuestro caso existe debido a la práctica común del Congreso colombiano que son los reemplazos, por lo que es necesario aplicar modificaciones al modelo para corregir los sesgos en las estimaciones, debido a esos retiros no aleatorios. El criterio adoptado para corregir potenciales sesgos, por retiros informativos, es el de la modelación conjunta de la información longitudinal con el tiempo hasta el 
retiro de los miembros del comité. Por considerar la estructura tiempo-dependiente de la información, en nuestra propuesta de modelo, consideramos que mejora la de Chappell et al. (2005). Acompañamos el modelo con un análisis descriptivo para poner en contexto el problema.

\section{Análisis descriptivo}

Las Comisiones Económicas contemplan 85 parlamentarios, pero debido a los reemplazos ocurridos durante el curso de las sesiones, hay un promedio de 87 asistiendo a las sesiones por año. Por ejemplo, en 2003, hubo 11 reemplazos con tres en la mitad del año y, por tanto, hubo 88 parlamentarios participando en las sesiones de ese año. Como 46 congresistas participaron en ambos periodos legislativos, entonces 124 fueron los que debieron participar en total en las comisiones, pero debido a la alta tasa de reemplazos fueron 189 los integrantes. Esto es 65 más de lo presupuestado. Lo que significa que el $34 \%$ de los dueños de una curul fueron reemplazados en algún momento. En total, hubo 49 sesiones donde los 189 parlamentarios hicieron 1.452 intervenciones, para un promedio de casi 6 sesiones por año y 30 intervenciones por sesión.

La Tabla 1 muestra la distribución de las covariables a lo largo del tiempo, sobre la que es im- portante mencionar tres aspectos para una buena comprensión: a) las distribuciones para el total del período 2002-2009 no son el promedio de las distribuciones por año, son las distribuciones de los 189 parlamentarios que participaron en todo el período; b) las distribuciones de las covariables dependientes del tiempo, para estos 189 , no aparecen porque no tienen ningún sentido; $\mathrm{y}$ c) por simplicidad se omite la información de un nivel en la distribución de cada variable. Las distribuciones para los 189 nos dicen que el $29,6 \%$ de los parlamentarios pertenecían a los partidos de la oposición; hubo más parlamentarios sin educación universitaria $(15,9 \%)$ que economistas (12,2\%); el $11 \%$ provenía de Bogotá, el 30,7\% del Caribe y el 58,3\% de las otras regiones; $y$, como ya se mencionó, el $27 \%$ fue condenado o tiene procesos judiciales abiertos. Respecto de las evoluciones en el año, señalamos para el partido que en 2002 hubo una participación de 35 (38,9\%) parlamentarios de oposición y $65(61,1 \%)$ de la coalición de gobierno; para los siguientes años del primer periodo legislativo la distribución fue muy similar, pero en el segundo baja considerablemente la oposición, teniendo para el 2006 una proporción de $25,9 \%$.

Variable dependiente: aproximadamente 17 parlamentarios participan en promedio por sesión, representando el $20 \%$ de los 87 que en promedio

\begin{tabular}{|c|c|c|c|c|c|c|c|c|c|}
\hline \multirow[t]{2}{*}{ Año } & \multirow{2}{*}{$\begin{array}{l}\text { Número de } \\
\text { congresistas }\end{array}$} & \multirow{2}{*}{$\begin{array}{c}\begin{array}{c}\text { Partido } \\
\text { Político }\end{array} \\
\text { Oposición }\end{array}$} & \multirow{2}{*}{\begin{tabular}{|c|}
$\begin{array}{c}\text { Proceso } \\
\text { Judicial }\end{array}$ \\
$\begin{array}{c}\text { Condenado o } \\
\text { con proceso }\end{array}$ \\
\end{tabular}} & \multirow{2}{*}{\begin{tabular}{|c|} 
Ponente \\
No Ponente
\end{tabular}} & \multirow{2}{*}{\begin{tabular}{|l|} 
Reemplazo \\
$\begin{array}{l}\text { Dueño de } \\
\text { una Curul }\end{array}$ \\
\end{tabular}} & \multicolumn{2}{|c|}{ Región } & \multicolumn{2}{|c|}{ Profesión } \\
\hline & & & & & & Bogotá & Caribe & Economista & $\begin{array}{c}\text { No } \\
\text { Profesional }\end{array}$ \\
\hline \multicolumn{10}{|c|}{ Primer Periodo Legislativo } \\
\hline 2002 & 90 & 38,9 & 31,1 & 51,1 & 94,4 & 10,0 & 28,9 & 16,6 & 13,3 \\
\hline 2003 & 88 & 37,5 & 26,2 & 59,1 & 87,5 & 11,4 & 27,3 & 12,5 & 17,0 \\
\hline 2004 & 85 & 36,5 & 28,2 & 70,6 & 91,8 & $\mathrm{H1}, 8$ & 25,9 & 14,2 & 13,1 \\
\hline 2005 & 87 & 37,9 & 27,5 & 50,6 & 81,6 & 11,5 & 29,9 & 16,1 & 19,5 \\
\hline \multicolumn{10}{|c|}{ Segundo Periodo Legislativo } \\
\hline 2006 & 85 & 25,9 & 36,4 & 72,9 & 100 & 10,6 & 31,8 & 16,5 & 2,4 \\
\hline 2007 & 85 & 25,9 & 31,7 & 41,2 & 90,6 & 9,4 & 30,6 & 12,9 & 5,9 \\
\hline 2008 & 88 & 24,7 & 30,7 & 47,2 & 87,5 & 10,2 & 30,7 & 13,5 & 7,9 \\
\hline 2009 & 88 & 26,1 & 20,5 & 29,5 & 79,5 & 12,5 & 28,4 & 12,5 & 6,8 \\
\hline $\begin{array}{l}02 / \\
09\end{array}$ & 189 & 29,6 & 26,9 & - & - & 11,0 & 30,7 & 12,2 & 15,9 \\
\hline
\end{tabular}

Fuente: elaboración propia basada en datos de Salinas (2015). 


\begin{tabular}{|c|c|c|c|}
\hline Año & $\begin{array}{c}\text { Coalición de } \\
\text { Gobierno }\end{array}$ & Oposición & Total \\
\hline \multicolumn{4}{|c|}{ Primer Periodo Legislativo } \\
\hline 2002 & 18,2 & 28,6 & 22,2 \\
\hline 2003 & 7,3 & 21,2 & 12,5 \\
\hline 2004 & 9,3 & 25,8 & 15,3 \\
\hline 2005 & 5,6 & 27,3 & 13,8 \\
\hline \multicolumn{4}{|c|}{ Segundo Periodo Legislativo } \\
\hline 2006 & 23,8 & 40,9 & 28,2 \\
\hline 2007 & 19,0 & 54,5 & 28,2 \\
\hline 2008 & 10,4 & 22,7 & 13,5 \\
\hline 2009 & 13,8 & 30,4 & 18,2 \\
\hline
\end{tabular}

por año pertenecen a las Comisiones Económicas. Sin embargo, solo 4 proponen debates: aproximadamente el 5\% de los 87 . El 31,7\% de los parlamentarios, que pasaron por las Comisiones Económicas, participaron al menos en un debate en todo el período de análisis. Las proporciones son $39,3 \%$ para los partidos de oposición y $28,6 \%$ para la coalición de gobierno. La Tabla 2 muestra estas proporciones a lo largo del tiempo, donde es evidente que los partidos de la coalición debaten más al principio que al final de cada período legislativo. La oposición mantiene un alto nivel de debate, con un pico en 2006 y 2007, cuando se discutió el Tratado de Libre Comercio (TLC) con Estados Unidos.

Suponemos que hay limitaciones dadas por el nivel educativo y la profesión para un adecuado análisis del Presupuesto, y que eso indica que las decisiones se tomarían sin plena racionalidad técnica. Para ampliar este aspecto, las tasas de debate (\# debates / \# parlamentarios) de la Tabla 3 se desglosan de acuerdo con los niveles de la variable "Profesión". Ciertamente, se observa que esta variable está relacionada con el debate.

La Tabla 3 también muestra que la tasa general de debate es de 1,2 por persona, y que los niveles de variables con menos tendencia al debate son mujeres, miembros de la coalición, reemplazos, no economistas, aquellos con problemas judiciales y provenientes de la región del Caribe.

En cuanto al tema de las intervenciones, los economistas las dedicaron en un $74 \%$ a tratar problemas macroeconómicos (como la deuda externa, el déficit presupuestario, etc.) y los no economistas solo en un 38\%. La oposición dedicó a los temas económicos un $60,6 \%$, y a los regionales y de vías y transporte un $6,8 \%$, mientras que esos valores para la coalición son de 39,2 y $25,0 \%$, respectivamente. En Salinas y Huertas (2018) se puede ver este aparte un poco más amplio.

\section{Modelo longitudinal}

En una sesión tipo se tiene información por parlamentario de si debatió o no en contra del Presupuesto. Variable binaria que desea explicarse con covariables como sexo, partido político o región, entre otras. Esto encaja, para ser analizado, con modelos de efecto fijo como la regresión logística, pero la situación es más compleja porque se trata de información de una sesión de 49. Así, la variable dependiente, por cada parlamentario, consiste en una secuencia de Unos (debates) y Ceros, que irá acompañada de información de covariables fijas como el "Sexo", y dependientes del tiempo como el "Reemplazo". Para este tipo de información, existen modelos denominados, de forma general, como modelos estadísticos longitudinales con respuesta binaria. Uno de ellos es el modelo con efectos aleatorios.

Una buena apreciación de la diferencia entre modelos de efecto fijo y aleatorio, establecida de forma particular para el caso de respuesta binaria, se puede ver en Hedeker y Gibbons (2006): 
Cuando las respuestas binarias se agrupan como cuando se miden repetidamente aspectos dentro de individuos, o se agrupan dentro de algunos estratos, el modelo de regresión logística de efectos fijos falla en sus suposiciones para caracterizar con precisión la dependencia en los datos. El modelo de efectos fijos supone que las observaciones son independientes y en este caso no lo son por cuanto están agrupadas dentro de individuos. Los modelos con efectos aleatorios permiten incorporar la correlación entre las mediciones agrupadas en los procesos de estimación. Se puede conceptualizar los efectos aleatorios como la representación de las diferencias específicas de los sujetos en la propensión a responder a lo largo del tiempo. Aquellos sujetos con mayor propensión a la respuesta exhibirán una mayor probabilidad de una respuesta positiva, condicionada a sus valores de los efectos fijos incluidos en el modelo (por ejemplo, un grupo tratamiento) (p. 155).

En nuestro caso, la respuesta o debate en contra del Presupuesto está agrupada entre individuos (legisladores) a lo largo del tiempo, y será modelada con una parte fija común a todos los individuos y una aleatoria o específica para cada uno. Si las covariables no son tiempo dependientes se consideran de efecto fijo y no tendrán parte aleatoria en el modelo.

Para establecer la notación, sea $i=1, \ldots, n$ las unidades de nivel 2 (individuos) y $j=1, \ldots, n_{i}$ las unidades de nivel 1 (observaciones anidadas). Sea $Y_{i j}$ el valor de la variable respuesta dicotómica asociada con la unidad $j$ de nivel 1 anidada dentro de la unidad $i$ de nivel 2. En nuestro caso, la respuesta del parlamento $i$ en la sesión $j$. El modelo de regresión logística se escribe en términos del logit de la probabilidad de una respuesta denotada $p_{i j}$. El modelo de interceptos aleatorios y su representación en términos de la latente subyacente $y$ son (ver Hedeker y Gibbons, 2006, p. 156):

$$
\begin{gathered}
\log \left(\frac{p_{i j}}{1-p_{i j}}\right)=x_{i j}^{T} \beta+v_{i} \\
y_{i j}=x_{i j}^{T} \beta+\sigma_{v} \theta_{i}+\varepsilon_{i j}
\end{gathered}
$$

donde $x_{i j}$ es un vector de covariables, $\beta$ es un

\begin{tabular}{|c|c|c|c|c|}
\hline & Economista & Otra Profesión & No Profesional & Total \\
\hline $\begin{array}{l}\text { Sexo } \\
\text { Hombre } \\
\text { Mujer }\end{array}$ & $\begin{array}{l}4,5 \\
1,0\end{array}$ & $\begin{array}{l}1,0 \\
0,7\end{array}$ & $\begin{array}{c}0,04 \\
0,0\end{array}$ & $\begin{array}{l}1,3 \\
0,7\end{array}$ \\
\hline $\begin{array}{l}\text { Partido Político } \\
\text { Coalición de Gobierno } \\
\text { Oposición }\end{array}$ & $\begin{array}{l}1,1 \\
9,6\end{array}$ & $\begin{array}{l}0,7 \\
1,5\end{array}$ & $\begin{array}{l}0,0 \\
0,1\end{array}$ & $\begin{array}{l}0,7 \\
2,6\end{array}$ \\
\hline $\begin{array}{l}\text { Proceso Judicial } \\
\text { Sin Proceso } \\
\text { Condenado o con Proceso }\end{array}$ & $\begin{array}{l}4,9 \\
3,0\end{array}$ & $\begin{array}{l}1,1 \\
0,7\end{array}$ & $\begin{array}{l}0,0 \\
0,2\end{array}$ & $\begin{array}{l}1,4 \\
0,9\end{array}$ \\
\hline $\begin{array}{l}\text { Reemplazo } \\
\text { Titular de una Curul } \\
\text { Reemplazo de una Curul }\end{array}$ & $\begin{array}{l}5,2 \\
0,5\end{array}$ & $\begin{array}{l}1,2 \\
0,1\end{array}$ & $\begin{array}{l}0,1 \\
0,0\end{array}$ & $\begin{array}{l}1,6 \\
0,1\end{array}$ \\
\hline $\begin{array}{c}\text { Región } \\
\text { Bogotá } \\
\text { Caribe } \\
\text { Otras }\end{array}$ & $\begin{array}{l}14,8 \\
2,8 \\
1,0\end{array}$ & $\begin{array}{l}1,9 \\
0,2 \\
1,2\end{array}$ & $\begin{array}{l}- \\
0,1 \\
0,0\end{array}$ & $\begin{array}{l}5,7 \\
0,4 \\
1,0\end{array}$ \\
\hline $\begin{array}{l}\text { Ponente } \\
\text { Ponente (de modificaciones) } \\
\text { No Ponente }\end{array}$ & $\begin{array}{l}6,1 \\
0,4\end{array}$ & $\begin{array}{l}1,1 \\
0,2\end{array}$ & $\begin{array}{l}0,1 \\
0,0\end{array}$ & $\begin{array}{l}1,6 \\
0,2\end{array}$ \\
\hline Total & 4,4 & 1,0 & 0,04 & 1,2 \\
\hline
\end{tabular}
vector de parámetros de regresión desconoci- 
dos, y $v_{i}$ es el efecto aleatorio para el que se asume una distribución $\mathrm{N}\left(0, \sigma^{2}\right)$. En forma estandarizada, los efectos aleatorios se representan como $v_{i}=\sigma_{v} \theta_{i}$, con $\theta_{i}$ distribuido $\mathrm{N}(0,1)$. Suponiendo que las observaciones dentro de un sujeto son independientes dados los efectos aleatorios, tenemos la siguiente verosimilitud observada para estimar los parámetros del modelo:

$L\left(\beta, \sigma_{v}^{2}\right)=\prod_{i=1}^{n} \int_{\theta} \prod_{i=1}^{n_{i}} \Psi\left(x_{i j}^{T} \beta+\sigma_{v} \theta_{i} \mid \beta\right)^{Y_{i j}}\left[1-\Psi\left(x_{i j}^{T} \beta+\sigma_{v} \theta_{i} \mid \beta\right)\right]^{1-Y_{i j}} g\left(\theta \mid \sigma_{v}^{2}\right) d \theta$

donde $\Psi$ representa la función de distribución acumulativa logística y $g(\theta)$ la distribución de los efectos aleatorios, es decir, $\mathrm{N}\left(0, \sigma^{2} v\right)$. De acuerdo con Lalonde, Nguyen, Yin, Irimata, y Wilson (2013), no hay muchos modelos adecuados para respuestas binarias en el tiempo, cuando los datos incluyen la correlación entre la respuesta y las covariables dependientes del tiempo. Uno de los inconvenientes es que la forma funcional marginal, cuando se integra sobre la distribución de los efectos aleatorios en (2), ya no tiene una forma logística. Aunque hay modelos recientes que dan soluciones a este problema como el de Parzen et al. (2011), adoptamos el modelo descrito anteriormente: no estamos interesados en modelos marginales. Nuestro propósito es determinar, en general, la relación entre los debates contra el Presupuesto y las características de los parlamentarios a lo largo del tiempo.

Para extender el modelo a múltiples efectos aleatorios, sea $z_{i j}$ el vector de variables de efectos aleatorios, y sea $v_{i}$ un vector de media o y matriz de varianza y covarianzas $\Sigma_{v}$. De forma estandarizada, los efectos aleatorios se representan como $v_{i}=T \theta_{i}$, donde $T T^{T}=\Sigma_{v}$. El modelo ahora se escribe como:

$$
\log \left(\frac{p_{i j}}{1-p_{i j}}\right)=x_{i j}^{T} \beta+z_{i j}^{T} T \theta_{i}
$$

Para una representación multinivel (modelo jerárquico logístico) de un modelo simple con una sola covariable en el nivel $1 x_{i j}$ y una variable en el nivel $2 x_{i}^{\prime}$ (de efecto fijo), tenemos que el nivel 1 en términos de logit y la variable latente subyacente $y$ son (ver Hedeker y Gibbons, 2006, p. 160):

$$
\begin{gathered}
\log \left(\frac{p_{i j}}{1-p_{i j}}\right)=\beta_{0 i}+\beta_{1 i} x_{i j} \\
y_{i j}=\beta_{0 i}+\beta_{1 i} x_{i j}+\varepsilon_{i j} .
\end{gathered}
$$

Suponiendo que $x_{i j}$ es una variable de efectos aleatorios, el modelo del nivel 2 es entonces:

$$
\begin{gathered}
\beta_{0 i}=\beta_{0}+\beta_{20} x_{i}^{\prime}+v_{0 i} \\
\beta_{1 i}=\beta_{1}+\beta_{21} x_{i}^{\prime}+v_{1 i} .
\end{gathered}
$$

Un modelo de interceptos y pendientes aleatorias con un efecto fijo más interacción, y reparametrización en las ecuaciones combinadas, para formar una sola ecuación en términos de la variable latente subyacente $y$ es:

$$
y_{i j}=\left(\beta_{0}+v_{0 i}\right)+\left(\beta_{1}+v_{1 i}\right) x_{i j}+\beta_{2} x_{i}^{\prime}+\beta_{3} x_{i}^{\prime} x_{i j}+\varepsilon_{i j}
$$




\subsection{Modelo conjunto para tratar retiros informativos}

Una investigación que genera medición longitudinal de alguna característica, en un período dado, puede tener retiros de unidades estadísticas que no permiten su medición completa en el período de estudio. Esta situación puede generar sesgos en los modelos estimados cuando dichos retiros son informativos (no al azar), lo que también se conoce como mecanismo de no respuesta, no ignorable (Little, 1993). En nuestro caso, los reemplazos, la no reelección y el abandono de las comisiones por parte de parlamentarios con problemas legales, generan cadenas de información muy cortas para caer en el problema de retiros informativos citado.

Una forma de tratar los retiros informativos es modelar el proceso longitudinal con algún factor asociado al mecanismo de no respuesta (digamos T); que, si corresponde al tiempo de retiro del estudio, se obtiene un caso particular de los denominados modelos conjuntos de información longitudinal y tiempo de supervivencia a un evento de interés (Tsiatis y Davidian, 2004). El modelo (1) o (3) para $Y$ se ajustará conjuntamente con el modelo para $T$, al que le escogimos uno $\log$-lineal: $\log \left(T_{i}\right)=\gamma X_{\mathrm{i}}+\sigma_{\mathrm{s}} w_{\mathrm{i}}$, con $\gamma$ un vector de parámetros (incluido el intercepto). Asociado a un vector de covariables explicativas $X_{i}$ y con $\sigma_{s}$ un parámetro de escala asociado a los errores $w_{i}$. El vector de covariables asociado a $T$ puede tener componentes comunes a los de $Y$. Si se supone a $w_{i}$ con distribución normal entonces, $T$ se distribuye log-normal (condicional en las covariables); y si $w_{i}$ se supone con distribución gumbel estándar, entonces $T$ se distribuye Weibull. Si el tiempo de supervivencia $T_{i}$ para el sujeto $i$ no se observa al finalizar el estudio (retiro en nuestro caso), entonces se dice que está censurado a la derecha por $C_{i}$, que se supone independiente de $T_{i}$. Los datos observados sobre el proceso del tiempo de supervivencia consisten en un par de variables aleatorias $\left(D_{i}, \delta_{i}\right)$, donde $D_{i}=\min \left\{T_{i{ }^{-}}\right.$ $C_{i}$ y y $\delta_{i}=\mathrm{I}\left(T_{i} \leq C_{i}\right)$, con $\delta_{i}$ indicando si $D_{i}$ es un valor no censurado de $T_{i}$.

El procedimiento de estimación comienza por determinar cómo se factoriza la densidad conjunta de $Y$ y $T$. Los "modelos de selección" (selection models) factorizan la densidad conjunta de $T$ y $Y$ como $f(t, y)=f(t \mid y) f(y)$, y los "modelos de mezcla" (mixture models) como $f(t, y)=f(y \mid t) f(t)$. Aunque los modelos de mezcla se usan principalmente para estudios longitudinales con retiro informativo (Hogan y Laird, 1997), no elegimos la estrategia para modelar nuestro problema porque preferimos corregir los sesgos sin incluir directamente el mecanismo de datos faltantes dentro del modelo longitudinal. Específicamente usaremos un modelo que liga el longitudinal con el mecanismo de no respuesta por medio de efectos aleatorios (random effects selection model). A través de ellos, el modelo de $T$ induce en el de $Y$ información sobre la censura informativa. Asumiendo que condicional en los efectos aleatorios, $T$ y $Y$ son independientes, la verosimilitud observada con variable dependiente binaria es:

$$
\begin{gathered}
L\left(\beta, \gamma, \sigma_{v}, \sigma_{s}\right)=\prod_{i=1}^{n} \int_{\theta} \prod_{j=1}^{n_{i}} \Psi\left(x_{i j}^{T} \beta+\sigma_{v} \theta_{i} \mid \beta\right)^{Y_{i j}}\left[1-\Psi\left(x_{i j}^{T} \beta+\sigma_{v} \theta_{i} \mid \beta\right)\right]^{1-Y_{i j}} f\left(d_{i}, \delta_{i} \mid x_{i}, \theta_{i}, \gamma, \sigma_{s}\right) g\left(\theta \mid \sigma_{v}\right) d \theta \\
f\left(d_{i}, \delta_{i} \mid x_{i}, \theta_{i}, \sigma_{S}\right)=f_{T}\left(d_{i} \mid x_{i}, \theta_{i}, \sigma_{s}\right)^{\delta_{i}} S_{T}\left(d_{i} \mid x_{i}, \theta_{i}, \sigma_{s}\right)^{1-\delta_{i}}
\end{gathered}
$$

con $S$ la función de supervivencia de $T$. La mayoría de propuestas de modelación conjunta están diseñadas para modelos con respuesta longitudinal continua y con aplicación de algoritmos EM para maximizar la verosimilitud conjunta. Muchas de ellas como una extensión de la propuesta de Wulfsohn y Tsiatis (1997). Tales algoritmos EM no son directamente aplicables cuando la respuesta es binaria, por tanto, necesitan ser modificados (Choi, Cai, Zeng y Olshan, 2013). Las propuestas de estimación, en este caso, son pocas y no están disponibles en paquetes estadísticos. Una estimación "fácil" para modelar respuesta binaria conjuntamente con el tiempo, al retiro del estudio, se puede hacer como una extensión de la propuesta de Guo y Carlin (2004), que maximiza de forma directa la log-verosimilitud del modelo con- 
junto con el procedimiento NLMIXED del software SAS (SAS Institute, 2005). Debido a su fácil aplicación mediante la adaptación de códigos de programación dispuestos en la web, recientemente se ha utilizado bastante para ajustar modelos conjuntos. Si en este procedimiento se ignoran los efectos aleatorios que enlazan los modelos longitudinal y de supervivencia, las estimaciones para el modelo longitudinal coinciden con las del procedimiento GLIMMIX del SAS, desarrollado para adaptarse a modelos lineales mixtos generalizados. Para los cuales, el modelo jerárquico logístico es un caso particular.

\subsection{Modelo ajustado}

Para nuestra propuesta solo incluimos como covariables, en el modelo del tiempo al retiro, a los efectos aleatorios, y en el modelo de la respuesta longitudinal (Debate) se incluyen las covariables: Partido Político (PP), Región (Reg), Profesión (Pf) y Reemplazo (Rp). El modelo conjunto (con la parte longitudinal en términos de la variable latente subyacente $y$ ) es entonces:

$$
\begin{gathered}
y_{i j}=\left(\beta_{0}+v_{0 i}\right)+\left(\beta_{1}+v_{1 i}\right) R p_{i j}+\beta_{2} P P_{i}+\beta_{3} R e g_{i}+\beta_{4} P f_{i}+\varepsilon_{i j} \\
\log \left(T_{i}\right)=\gamma_{0}+\gamma_{1} v_{0 i}+\gamma_{2} v_{1 i}+\sigma_{s} w_{i}
\end{gathered}
$$

donde $\gamma_{1}$ y $\gamma_{2}$ son parámetros de ruido, y $v_{o i}$ y $v_{1 i}$ se tratan como parámetros a estimar en el modelo para $Y$ y como covariables en el modelo para $T$. El tiempo hasta el retiro se analizó con un modelo de vida acelerada con distribución de Weibull. La estimación del modelo en (8) se da en la Tabla 4, que no muestra la estimación de $\gamma_{2}$ porque es casi cero. El parámetro $\sigma^{2}{ }_{v}$ mide la variabilidad entre sesiones, su valor estimado en relación con su error estándar estimado, indica que hay un efecto de la sesión en los debates, es decir, la cantidad de debates difiere entre sesiones. Este resultado justifica el uso del modelo de efectos mixtos (fijos y aleatorios). La significancia en el parámetro de ligadura $\gamma_{1}$, da una clara indicación de que el patrón de retiros no es aleatorio, validando el uso del modelo conjunto.

A partir de los signos de los coeficientes del modelo estimado, podemos concluir que si el legislador pertenece a los partidos de oposición, representa a la capital del país, es economista y es titular de una curul (no es reemplazo), es más probable que debata en contra del Presupuesto. Los parámetros estimados indican que el chance de debate: a) de los miembros de la oposición es 2,4 veces el de los miembros de la coalición de gobierno; b) de los economistas es 40 veces el de los no profesionales y 2,6 veces el de los no economistas; c) de los parlamentarios que tienen una curul es casi 4 veces el de los parlamentarios que reemplazan a otros; y d) de los parlamentarios de Bogotá es casi 6 veces el de los del Caribe y 2,4 veces el que tienen otras regiones.

Dada la alta tasa de debate para los hombres y baja para las mujeres (ver Tabla 3), podría esperarse que la variable fuera significativa para explicar el Debate. Sin embargo, en presencia de otras variables significativas el efecto del sexo se diluye en el modelo. Que las mujeres no participen en muchos debates se debe a que tienen perfiles proclives al no debate, como no ser economistas, venir de la región del Caribe y pertenecer a la coalición de gobierno: de las 23 mujeres que pasaron por las Comisiones Económicas, el $96 \%$ no son economistas, el $40 \%$ proviene de la región del Caribe y el $83 \%$ pertenece a partidos de la coalición de gobierno.

La variable "Ponente" no es significativa para explicar el Debate en un modelo simple (valor- $p=$ 0,473). Tampoco lo es en todos aquellos donde la variable Región no esté incluida. La variable Ponente resulta significativa cuando la Región también está incluida en el modelo; ello debido a la 
alta asociación que muestran las dos variables (se rechaza una prueba de independencia entre variables, valor- $p<0,0001)$. Este es un claro ejemplo de una relación entre dos variables debidas a un tercer efecto y, por tanto, no fue incluida en el modelo final de la Tabla 4.

El "Proceso Judicial" explica el Debate en un modelo simple (valor $-p=0,003)$, pero no lo explica cuando la Región está en el modelo, debido a la relación existente entre estas dos variables independientes (se rechaza una prueba de independencia entre variables, valor- $p<0,0001$ ). $\mathrm{El}$ "Proceso Judicial" es significativo si se incluye en lugar de la variable Región en el modelo (8) (valor- $p=0,007$ ). En este caso, el chance de debate para los miembros del parlamento sin procesos judiciales es 1,6 veces el de los condenados o con procesos judiciales abiertos, es decir, $60 \%$ más grande.

\section{Discusión}

Del análisis descriptivo se concluye que hay situaciones indeseables para los parlamenta- rios, que pueden influir negativamente en una decisión técnica adecuada del Presupuesto: hubo una cantidad excesiva de reemplazos sin ninguna razón técnica justificable y también una cantidad excesiva de parlamentarios condenados o con procesos judiciales abiertos. Otra situación sugerida como no apropiada es que hubo en las Comisiones Económicas más miembros sin profesión que economistas. Puntualizamos el resultado pues los parlamentarios reemplazantes, los parlamentarios con problemas judiciales y los parlamentarios sin educación profesional, prácticamente no hablan en las sesiones y, cuando lo hacen, su contribución es irrelevante para las decisiones sobre el Presupuesto (Salinas y Huertas, 2018). Es claro concluir, con base en estas características, que el Debate se ve modificado por sesgos inducidos a través de variables como el Proceso Judicial, y también por las limitaciones que dan variables como la Profesión y el Reemplazo. Al respecto, tenemos las siguientes observaciones sobre las variables explicativas del Debate, en orden de importancia dentro del

\begin{tabular}{|c|c|c|c|c|c|c|}
\hline \multicolumn{7}{|c|}{$\begin{array}{c}\text { Tabla 4. Modelo conjunto para "el Debate en Contra del Presupuesto" } \\
\text { y el tiempo al retiro de las Comisiones Económicas }\end{array}$} \\
\hline \multicolumn{2}{|c|}{ Efecto } & Estimación & $\exp (\hat{\beta})$ & Error Estándar & t-valor & $\operatorname{Pr}>|t|$ \\
\hline \multicolumn{7}{|c|}{ Solución para efectos fijos longitudinales } \\
\hline Intercepto & $\left(\beta_{0}\right)$ & $-8,470$ & - & 1,121 & $-7,9$ & $<, 0001$ \\
\hline Partido Político & (B) & 0,886 & 2,4 & 0,151 & 5,8 & $<, 0001$ \\
\hline \multicolumn{7}{|l|}{ Región } \\
\hline Bogotá & $\left(\beta_{2}\right)$ & 1,775 & 5,9 & 0,235 & 7,5 & $<, 0001$ \\
\hline Otras & $\left(\beta_{22}\right)$ & 0,880 & 2,4 & 0,217 & 4,0 & $<, 0001$ \\
\hline Caribe & & r. & . & . & . & . \\
\hline \multicolumn{7}{|l|}{ Profesión } \\
\hline Economista & $\left(\beta_{3}\right)$ & 3,688 & 40,0 & 1,004 & 3,8 & 0,0002 \\
\hline Otra Profesión & $\left(\beta_{32}^{3}\right)$ & 2,744 & 15,2 & 0,998 & 2,9 & 0,0060 \\
\hline No-Profesional & & 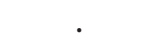 & 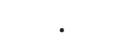 & & . & . \\
\hline Reemplazo & $\left(\beta_{4}\right)$ & 1,428 & 4,2 & 0,516 & 1,9 & 0,0057 \\
\hline \multicolumn{7}{|c|}{ Parámetros de covarianza para el modelo longitudinal } \\
\hline Intercepto & $\left(\sigma_{v}\right)$ & 0,486 & - & 0,198 & & \\
\hline \multicolumn{7}{|c|}{ Solución para el tiempo al evento } \\
\hline Intercepto & $\left(\gamma_{0}\right)$ & 1,638 & - & 0,053 & 29,6 & $<, 0001$ \\
\hline & $(\gamma)$ & 0,738 & - & 0,303 & 3,2 & 0,015 \\
\hline Escala & $(\sigma s)$ & 0,168 & - & 0,070 & & \\
\hline
\end{tabular}


modelo longitudinal:

Región. Se puede resumir la discusión sobre el Presupuesto respecto a la Región, por el desdén mostrado por los parlamentarios del Caribe en el debate, por el interés mostrado por los parlamentarios de Bogotá sobre el diseño del Presupuesto, desde el punto de vista macroeconómico, y por el constante reclamo de recursos para sus regiones que hicieron los parlamentarios del resto del país. Adicional a su relación con la variable de problemas judiciales, podemos ver que la Región tiene muchos matices que la convierten en la más importante para explicar la variable Debate.

Partido Político. La coalición debate menos que la oposición y lo hace más al comienzo de los períodos legislativos que al final, sin ninguna razón técnica aparente. Como la coalición, por ser mayoría, termina aprobando el proyecto de Presupuesto, podría suponerse que existe alguna estrategia consistente en oponerse, al inicio, al proyecto y presionar al gobierno para obtener algún beneficio político; pero es una apreciación que no se puede demostrar con base en la información disponible. De todas formas, lo importante del resultado es la tendencia especial del debate no atribuible a aspectos técnicos. Que la política influye en el proceso de toma de decisiones sobre el Presupuesto es un resultado que no sorprende. En ese mismo sentido, Giraldo (2009) concluye que la naturaleza del Presupuesto es de orden político.

Profesión. Varias cifras inducen a concluir que los legisladores no economistas debaten menos que los economistas. A las ya citadas sumamos otra contundente: el $43 \%$ de los debates lo hace el $12 \%$ de parlamentarios que tiene instrucción superior en economía. Aunque la falta de capacitación profesional en economía puede ser asistida por un grupo asesor proporcionado por la nación, según el resultado anterior, aparentemente este grupo no está haciendo un asesoramiento adecuado. Para esta apreciación y, en general, para la conclusión del artículo, asumimos que la discusión, al interior de las comisiones, debe estar implícita en el trabajo de los parla- mentarios, pues el Congreso es el escenario por excelencia donde se "discuten" y se generan las reformas que necesita la nación. Además, una de las funciones del Congreso es el control político: para eso también se le elije. Por lo tanto, es lógico que los parlamentarios ejerzan su labor debatiendo y sería contradictorio tener a un Congreso haciendo control político en silencio.

Reemplazo. Los reemplazos se han constituido en una costumbre política en Colombia como una estrategia de compartir el poder entre los diferentes líderes regionales, y a pesar de tener relación con la Región y el Partido Político, no es tan fuerte como para que termine saliendo del modelo para el Debate. Como no es de esperarse que algo tan complejo como el Presupuesto se domine llegando en medio de las sesiones, es muy grande el impacto negativo que tienen los reemplazos para el buen entendimiento del Presupuesto $y$, por ende, a la contribución para sus modificaciones.

Proceso Judicial. Aunque no queda en el modelo por su relación con la Región, de todas formas es un aspecto que no debe desconocerse como factor asociado en la determinación del Presupuesto, que necesita un análisis especial desde el criterio de la ciencia política (no abordado en este trabajo). De todas formas, es importante señalar que no hay un caso similar, en la historia democrática reciente del planeta, de tantos parlamentarios en ejercicio condenados y puestos en la cárcel.

No perdamos de vista que el propósito final del artículo no es modelar el debate per se. El modelo obtenido, en extensa deliberación, es una herramienta que nos permitirá decidir si hay factores no técnicos asociados a la determinación del Presupuesto y, de ahí, concluir que las decisiones no fueron tomadas en un ambiente puramente racional. Conclusión derivada de un escaso debate por desconocimiento del problema. Esto significa que habría sesgos y limitaciones en el decisor. Pero también debemos tener en cuenta que las limitaciones dadas por el precario nivel educativo, la baja instrucción superior en economía o el poco tiempo de permanencia 
en las comisiones debido a los reemplazos -y que conllevan a un escaso debate- no necesariamente implican bajo dominio del Presupuesto, para con ello concluir razonabilidad acotada en su determinación. Aunque existen evidencias para asumirlo así, veamos:

Con una mirada fuera del modelo, el no debate podría relacionarse con el dominio o no del Presupuesto. Por varias razones que incluyen, entre otras: a) los parlamentarios no dominan el tema del Presupuesto, pero como sólo se mueven por intereses particulares que igual alcanzan sin hacer debates, no usan los recursos del estado para poder entenderlo y debatirlo; b) a pesar de haber interés por parte de los parlamentarios en hacer un buen análisis del Presupuesto, sus limitaciones mencionadas no les permiten tener el adecuado entendimiento del tema, como para debatirlo, así usen debidamente los recursos del estado; c) los parlamentarios dominan el tema del Presupuesto, pero no lo debaten por limitaciones de oratoria o porque no les interesa hacerlo. Lo que a ellos les interesa es hacer los movimientos políticos necesarios para hacer las mejores modificaciones posibles al Presupuesto; y d) si es ingenuo creer que para hacer buenas políticas lo que necesitan los agentes decisores es buen conocimiento, entonces tendríamos una variación al punto (a): los parlamentarios dominan el tema del Presupuesto, pero no lo debaten debido a que alcanzan sus ambiciones personales sin la necesidad de hacerlo.

Con base en la información disponible no se puede concluir el porqué del escaso debate en la discusión planteada. Aun así, hay evidencias en el ambiente político que nos permiten inclinarnos más hacia alguna de las opciones anteriores. Empecemos por plantear que los debates le sirven a los parlamentarios para mostrar eficiencia a sus electores. Aquí es bueno señalar que en Salinas y Huertas (2018) se advierte que hay casos donde los debates no son atendidos por los integrantes de las comisiones ni por sus invitados. Son más una vitrina pensada para las cámaras de televisión. Con desatención o no, los debates dan al parlamentario liderazgo tanto popular como al interior del Congreso. Algo que no dudaría en aprovechar políticamente. Luego, si un parlamentario quiere hacer presión al gobierno para obtener algún rédito político o para obtener mejoras al Presupuesto, ¿por qué no hacerlo debatiendo en las comisiones? Sería muy efectivo y fructífero en todo sentido; no en vano la coalición hace bastantes debates al inicio de cada periodo legislativo, que es coincidente con el presidencial.

La opción (d) no se vería ilógica si el tema del Presupuesto fuera sencillo, que aún con experiencia en el tema debe estudiarse y analizarse cada año. Conocimiento con mucho detalle técnico que no se ve crucial a la hora de hacer acuerdos políticos. Difícil aceptar la opción (c) dado que el Presupuesto se aprueba con base en las mayorías de la coalición de gobierno, sin que se le hagan cambios fundamentales, a pesar de tener serias críticas técnicas (Salinas y Huertas, 2018). Efectivamente la variación entre el proyecto de Presupuesto y el finalmente aprobado no supera el $1 \%$, un triunfo de la política sobre el razonamiento económico. Ese bajo porcentaje de modificación podría explicarse por la rigidez existente en los presupuestos, pero no hay en las actas ninguna constancia, manifestada por los parlamentarios, sobre por qué aprueban forzosamente el Presupuesto debido al problema de rigidez. Adicionalmente, la oposición alega que la aprobación se consigue por medio de prebendas como los auxilios parlamentarios, y que reformas favorables no se hacen porque priman en el Congreso los intereses políticos y de carácter electoral.

Sobre lo anterior, vale la pena mirar el estudio de Newman y Ángel (2017), quienes señalan como segundo aspecto que favorece la corrupción al clientelismo con predominación local, cuya "función consiste en distribuir recursos en las localidades, bajo la intermediación de los caciques políticos ligados, a su turno, a los miembros del Congreso Nacional" (p. 21). Uno de los principales problemas de Colombia es la corrupción, situación verificable en el índice de percepción de corrupción establecido por Transparencia Internacional, que para 2017 lo fija en 37 (o es altamente corrupto y 100 muy limpio). Lejos de la media mundial calculada 
en 47 (Transparency International, 2017). No es de extrañar entonces que el Congreso tenga un altísimo nivel de desaprobación dentro de la población colombiana, que para el periodo de análisis de este estudio rondaba el $54 \%$ y para febrero de 2018 se encontraba en $84 \%$ (El País, 2017). Por tanto, en un desprestigiado Congreso, donde 71 de sus miembros terminaron presos (corrupción incluida) y donde se presentaron excesivos reemplazos como claro reflejo del clientelismo mencionado, es difícil asumir que la determinación del Presupuesto se realiza en un ambiente estrictamente técnico y racional, donde todos los agentes participan activa y debidamente con debates para tomar la mejor decisión y llegar al diseño óptimo.

\section{Conclusión}

Tenemos unos parlamentarios tanto de la oposición como de la coalición que dominan el Presupuesto y debaten bastante, teniendo en común el ser economistas. También hay una porción mayoritaria que no debate y no es economista, y como no sería lógico ubicarla dentro de las opciones que asumen dominio del Presupuesto, los casos de las opciones (c) y (d) serían poco frecuentes en las Comisiones Económicas (si es que existen). La no concreción de debates encuentra mucha explicación en las limitaciones mencionadas para los parlamentarios.

Un punto negativo adicional que impide concretar un análisis adecuado del Presupuesto, se encuentra en las asimetrías de información existentes en el proceso decisorio. Fundamentadas en las quejas de los parlamentarios por no tener la información completa, porque les llega a destiempo, por contradicción en algunas cifras registradas en el proyecto y porque, en general, estamos lejos del nivel de los países desarrollados, donde sí se tienen los instrumentos suficientes para hacer un análisis apropiado del Presupuesto (Salinas y Huertas, 2018). Para obtener información del gobierno, se acude incluso al derecho de petición. Por lo tanto, con base en las evidencias del modelo longitudinal y de las conclusiones de Salinas y Huertas (2018), tenemos el siguiente resultado:

El escaso debate sobre el proyecto de Presupuesto se explica por las limitaciones en su dominio por parte de los congresistas, como consecuencia de casos de bajo nivel educativo, baja instrucción superior en economía y baja permanencia en las comisiones debida a los reemplazos. Esto sumado a las asimetrías de información existentes en el proceso (Salinas y Huertas, 2018) y al sesgo político que otorgan el partido, la región y los procesos judiciales de los congresistas, nos permite concluir que las decisiones sobre el Presupuesto no se toman con una estricta racionalidad técnica y que hay evidencias de una racionalidad acotada en la toma de decisiones.

Como trabajo futuro, se tiene presupuestado ampliar la base de datos con la información de lo sucedido en los dos periodos de gobierno del presidente Juan Manuel Santos (2010-2018). En especial, para analizar la dinámica de los partidos políticos, debido a que en varios de ellos hay intercambio de roles en el tipo de oposición al gobierno rigente.

\section{Referencias bibliográficas}

Caamaño, J. y Lago-Peñas, S. (2011). Combining incrementalism and exogenous factors in analyzing national budgeting: an application to Spain. Public Finance Review, 39(5), 712-740. https://doi. org/10.1177/1091142111412578

Chappell, H. W., McGregor, R. R. \& Vermileya, T. (2005). Committee decisions on monetary policy. Evidence from Historical Records of the Federal Open Market Committee. Cambridge, Mass.: MIT Press. 
Choi, J., Cai, J., Zeng, D. \& Olshan A. (2013). Joint Analysis of Survival Time and Longitudinal Categorical Outcomes. Statistics in Biosciences, 7(1), 19-47. https://doi.org/10.1007/s12561-013-9091-z

Durlauf, E. D. \& Blume, L. E. (Eds.). (2008). The New Palgrave Dictionary of Economics. Second Edition. London, UK: Palgrave Macmillan.

Eijffinger, S. C., Mahieu, R. J. \& Raes, L. (2013). Estimating the preferences of central bankers: An analysis of four voting records. CEPR Discussion Paper, No. DP9602. Tilburg, Netherlands: Tilburg University, Center for Economic Research. http://dx.doi.org/10.2139/ssrn.2317678

El País. (2017). Encuesta Gallup Poll. Recuperado de https://www.elpais.com.co/especiales/encuestagallup-poll-123.pdf

Giraldo, C. (2009). Finanzas Públicas en América Latina: La economía política. Bogotá D.C., Colombia: Ediciones desde abajo.

Good, D. (2011). Still budgeting by muddling through: Why disjointed incrementalism lasts. Policy and Society, 30(1), 41-51. https://doi.org/10.1016/j.polsoc.2010.12.005

Guo, X. \& Carlin, B. P. (2004). Separate and Joint Modeling of Longitudinal and Event Time Data Using Standard Computer Packages. The American Statistician, (58), 16-24. https://doi. org/10.1198/0003130042854

Hansson, S. O. (1994). Decision theory. A brief introduction. Recuperado de http://fitelson.org/current/ hansson_decision_theory.pdf

Hedeker, D. \& Gibbons, R. (2006). Longitudinal Data Analysis. New Jersey, US: John Wiley \& Sons.

Hogan, J. \& Laird, N. M. (1997). Model-Based Approaches to Analyzing Incomplete Longitudinal and Failure Time Data. Statistical Methods in Medical Research, 16(1-3), 259-272. https://doi.org/10.1002/ (SICI)1097-0258(19970215)16:3<259::AID-SIM484>3.0.CO;2-S

Jung, A. \& Kiss, G. (2012). Preference heterogeneity in the EEC inflation-targeting countries. European Journal of Political Economy, 28(4), 445-460. http://doi.org/10.1016/j.ejpoleco.2012.05.004

Lalonde, T. L., Nguyen, A. Q., Yin, J., Irimata, K. \& Wilson, J. R. (2013). Modeling Correlated Binary Outcomes with Time-Dependent Covariates. Journal of Data Science, 11(4), 715-738. https://doi. org/10.6339/JDS.2013.11(4).1195

Lindblom, C. (1959). The Science of 'muddling through. Public Administration Review, 19(2), 79-88. https://doi.org/10.2307/973677

Little, R. C. (1993). Pattern-Mixture models for multivariate incomplete data. Journal for the American Statistical Association, 82(421) 125-134. https://doi.org/10.2307/2290705

Newman, V. y Ángel, P. (2017). Sobre la corrupción en Colombia: marco conceptual, diagnóstico y propuestas de política. Bogotá, Colombia: Fedesarrollo.

Parzen, M., Ghosh, S., Lipsitz, S., Sinha, D., Fitzmaurice, G. M., Mallick, B. K. \& Ibrahim, J. G. (2011). A generalized linear mixed model for longitudinal binary data with a marginal logit link function. Annals of Applied Statistics, 5(1), 449-467. https://doi.org/10.1214/10-AOAS390

Salinas, A (2015). Análisis de la Toma de Decisiones en Comités Económicos, el caso Colombiano de la Toma de Decisiones de las Comisiones Económicas del Senado y la Cámara de Representantes, 
en el periodo 2002-2009. Recuperado de http://www.bdigital.unal.edu.co/52654/1/ adrianafranciscasalinasesteban.2015.pdf

Salinas, A. y Huertas, J. (2018). Análisis de la Toma de Decisiones en las Comisiones Económicas del Congreso Colombiano, Usando Análisis Estadístico de Datos Textuales. Análisis Político, 31(92), 202-228. http://doi.org/10.15446/anpol.v31n92.71108

Salinas, E. y Jalil, A. (2014). Herramientas teórico-conceptuales para el análisis de la toma de decisiones en comités de economía pública. Análisis Político, 27(80), 165-190. http://dx.doi.org/10.15446/anpol. v27n80.45619

SAS Institute. (2005). SAS/STAT 9.1. Cary, North Caroline, US: SAS Institute Inc.

Simon, H. (1957). El comportamiento administrativo. Estudio de los procesos de adopción de decisiones en la organización administrativa. Ciudad de México, México: Aguilar.

Transparency International. (2017). Corruption Perceptions Index 2017. Recuperado de https://www. transparency.org/news/feature/corruption_perceptions_index_2017

Tsiatis, A. A. and Davidian, M. (2004). Joint Modeling of Longitudinal and Time-to-Event Data: An Overview. Statistica Sinica, (14), 809-834.

Wildavsky, A. (1964). Politics of the Budgetary Process. Boston, US: Little, Brown and Company.

Wulfsohn, M. \& Tsiatis, A. A. (1997). A Joint Model for Survival and Longitudinal Data Measured with Error. Biometrics, 53(1), 330-339. http://dx.doi.org/10.2307/2533118 